\title{
The Association between Substance Use Disorders and Long-Term Outcome of Stroke: Results from a Population-Based Study of Stroke among 450,229 Urban Citizens
}

\author{
Mohammad Sobhan Sheikh Andalibi ${ }^{a} \quad$ Amir Rezaei Ardani $^{b} \quad$ Amin Amiri ${ }^{c}$ \\ Negar Morovatdar ${ }^{d}$ Amirhossein Talebic, e Mahmoud Reza Azarpazhooh ${ }^{\mathrm{f}, \mathrm{g}, \mathrm{h}}$ \\ Naghmeh Mokhber ${ }^{\mathrm{i}}$ \\ anternational UNESCO Center for Health-Related Basic Sciences and Human Nutrition, Mashhad University of \\ Medical Sciences, Mashhad, Iran; 'bsychiatry and Behavioural Sciences Research Centre, Mashhad University of \\ Medical Sciences, Mashhad, Iran; 'Department of Neurology, Ghaem Hospital, Mashhad University of Medical \\ Sciences, Mashhad, Iran; ${ }^{d}$ Clinical Research Development Unit, Faculty of Medicine, Mashhad University of Medical \\ Sciences, Mashhad, Iran; 'Department of Neurology, Center of Expertise for Parkinson \& Movement Disorders, \\ Radboud University Medical Center, Nijmegen, The Netherlands; fDepartment of Clinical Neurological Sciences, \\ Western University, London, ON, Canada; ${ }^{9}$ Department of Epidemiology and Biostatistics, Schulich School of \\ Medicine \& Dentistry, Western University, London, ON, Canada; ${ }^{\mathrm{h}}$ Stroke Prevention and Atherosclerosis Research \\ Centre, Robarts Research Institute, Western University, London, ON, Canada; 'Department of Psychiatry, St Joseph's \\ Health Care London, Western University, London, ON, Canada
}

\section{Keywords}

Disability · Mortality · Population-based studies .

Substance use disorder · Stroke outcome

\begin{abstract}
Introduction: Little is known regarding long-term stroke outcomes in patients with substance use disorder (SUD). Based on anecdotal data, some individuals use illicit drugs, particularly opioids, in an attempt to reduce stroke mortality, disability, or recurrence. This study is aimed to assess the effect of SUD on stroke outcomes. Methods: Patients were recruited from the Mashhad Stroke Incidence Study, a population-based study of stroke in Iran. For a period of 1 year, all patients with first-ever stroke (FES) were recruited and then
\end{abstract}

followed up for the next 5 years. Disability and functional dependency were defined using modified Rankin Scale $(>2)$ and Barthel Index $(<60)$, respectively. We compared the cumulative rates of mortality in follow-up points using the logrank test. We used multivariable logistic, Cox regression and competing risk models to assess adjusted hazard ratio (aHR) with $95 \%$ confidence interval $(\mathrm{Cl})$ of stroke disability, functional dependency, mortality, and recurrence among those with a history of SUD. Results: 595 FES patients (mean age of $64.6 \pm 14.8$ years) were recruited in this study. Eighty-one (13.6\%) were current substance users, including opium ( $n=$ $68)$, naswar $(n=5,6.1 \%)$, hashish $(n=1)$, heroin $(n=1)$, and

M.S.S.A. and A.R.A. contributed equally. 
$(n=7)$ others. The frequency of vascular risk factors was similar between the SUD and non-SUD groups, except for a higher rate of cigarette smoking in the SUD group $(p<0.001)$. After adjusting for various sociodemographic variables, vascular risk factors, and the severity of stroke at admission, SUD increased the 3-month (aHR: 1.60, Cl: 1.01-2.49), 1-year (aHR: 1.73, Cl: 1.20-2.65), and 5-year (aHR: 1.72, Cl: 1.23-2.35) poststroke mortality risk. We did not observe a significant change in the risk of stroke recurrence, disability, and functional dependency in those with a history of SUD. Conclusion: SUD increased the hazard ratio of stroke mortality with no effect on the disability rate. The public should be advised about the potential harm of substance abuse.

(c) 2021 S. Karger AG, Base

\section{Introduction}

Substance use disorders (SUD) may be a direct or indirect risk factor for stroke [1-7]. The relationship between illicit drugs and stroke incidence, especially in young adults, has been reported in several studies $[1,2,8]$. However, little is known regarding long-term stroke outcome in substance users. Such information is crucial in public health and substance use prevention programs. First, the overall rate of SUD is on the rise worldwide [9]; therefore, it is likely there are patients with clusters of noncommunicable disease, vascular risk factors, and SUD in all countries. In addition, in some Asian traditional medicine, such as Chinese, Indian, and Iranian, narcotics are considered as a remedy with possible therapeutic effects [10-13]. Many people in Middle Eastern countries have this misconception that opium consumption may have protective effects against vascular risk factors (e.g., hyperlipidemia and diabetes) [14] and cerebro- or cardiovascular diseases [15].

In a large prospective population-based study of stroke, the Mashhad Stroke Incidence Study (MSIS), we reported an excessive stroke incidence in Iran compared with most Western countries, with stroke occurring at younger ages [16]. Unfortunately, a substantial number of our patients died $[17,18]$, remained disabled [19], or had stroke recurrences [20] during 5-year follow-up. A higher rate of stroke with poor outcomes in our study may be explained by differences in the rate of vascular risk factors [16] and etiology of stroke in our population [21, 22 ]. In addition, socioeconomic disadvantage [23], unhealthy lifestyle [24], and different socioeconomic variables [25], including high-risk behaviors (e.g., SUD), may be associated with stroke incidence and outcomes. The current study was designed to assess the association be- tween SUD and long-term outcomes of stroke, including mortality, stroke recurrence, disability, and functional dependency.

\section{Materials and Methods}

\section{Study Population}

Patients were recruited from the MSIS. The methods of the MSIS have been previously described elsewhere in detail [16]. In summary, during a 12-month period (2006-2007), we prospectively ascertained all potential cases with stroke in 3 health districts of Mashhad $(N=450,229)$, an urban area of Iran. We delineated the study area by the main boulevards in Mashhad. About 1,000 volunteers helped us to find stroke patients not admitted to the hospital. A group of stroke experts reviewed all potential cases before inclusion in the study. To identify mortality [17], stroke recurrence [20], poststroke disability, and functional dependency [19], we followed all cases at 5 points, including 1 week, 1 month, 3 months, 1 year, and 5 years after their index stroke. The MSIS is the first population-based cohort study of stroke in the Middle East. Our database includes a wide range of variables, including sociodemographic information, vascular risk factors, and type and etiology of stroke.

The study was conducted according to the Declaration of Helsinki guidelines and approved by the Ethics Committee of Mashhad University of Medical Sciences (No. 910678). Informed consent was obtained from each participant or the next of kin before any interview or neurologic examination.

\section{Definitions}

We defined stroke according to the World Health Organization [26]. The severity of stroke was defined based on the National Institutes of Health Stroke Scale (NIHSS) at admission and each follow-up session. Poststroke disability was assessed using modified Rankin Scale (mRS). The mRS measures the degree of poststroke disability in 6 different levels, ranging from 0 (lack of symptoms) to 5 (severe disability) and 6 indicating death. We used Barthel Index (BI) to measure functional dependency. The BI is an ordinal score, including 10 important questions regarding performance in activities of daily living. We defined disability as an $\mathrm{mRS}$ of $>2$ and functional dependency as a BI of $<60$ [19]. According to the patients' neighborhood, patients were categorized into 3 subgroups: less privileged area, middle privileged area, and high privileged area [23]. As a part of our clinical assessments, trained nurses and medical students at the residency level had a face-to-face interview regarding SUD, including opioids (burned and the sap), hashish, or similar compounds, heroin, amphetamine-type stimulants, hallucinogens, naswar (a mixture of tobacco and slaked lime, usually with some flavors such as indigo and juniper ash), and inhalants. We divided the patients into 2 groups: current substance users, including former users who had not reached sustained remission (SUD group), and lifelong nonsubstance users or former users who had reached sustained remission (non-SUD group). We used the Diagnostic and Statistical Manual of Mental Disorders 4th edition, text revision (DSM IV-TR) criteria to define the sustained remission in drug users. According to the DSM IV-TR criteria, one should continue complete abstinence for at least 1 year (12 consecutive months) to reach sustained remission [27]. 
Table 1. Demographic and socioeconomic characteristics of 595 cases with first-ever stroke with and without history of current SUD

\begin{tabular}{|c|c|c|c|}
\hline Parameters & $\begin{array}{l}\text { SUD group } \\
(n=82)\end{array}$ & $\begin{array}{l}\text { Non-SUD group } \\
(n=513)\end{array}$ & $p$ value \\
\hline Age, $\mathrm{yr}^{\dagger}$ & $63.5 \pm 16.0$ & $64.5 \pm 14.8$ & 0.57 \\
\hline Sex (male), $n(\%)$ & $58(70.7)$ & $250(48.7)$ & $<0.001$ \\
\hline Married, $n(\%)$ & $62(76.5)$ & $386(75.7)$ & 0.87 \\
\hline Low education (<12 yr), $n(\%)$ & $64(79.0)$ & $410(80.7)$ & 0.72 \\
\hline Low income, $n(\%)$ & $50(61.0)$ & $266(51.9)$ & 0.24 \\
\hline Children number & $5.0(3.0-8.0)$ & $5.0(3.0-6.0)$ & 0.40 \\
\hline Living on less privileged area, $n(\%)$ & $45(54.9)$ & $209(40.8)$ & 0.04 \\
\hline Body mass index, $\mathrm{kg} / \mathrm{m}^{2 \dagger}$ & $24.6 \pm 5.9$ & $25.6 \pm 5.5$ & 0.17 \\
\hline Waist circumference, $\mathrm{cm}^{*}$ & $94.5(86.2-100.0)$ & $96.0(87.0-105.0)$ & 0.12 \\
\hline Atrial fibrillation, $n(\%)$ & $4(5.1)$ & $46(9.2)$ & 0.22 \\
\hline Transient ischemic attack, $n(\%)$ & $3(3.7)$ & $40(7.8)$ & 0.18 \\
\hline Hypertension, $n(\%)$ & $43(52.4)$ & $309(60.6)$ & 0.16 \\
\hline Diabetes mellitus, $n(\%)$ & $20(24.4)$ & $156(30.5)$ & 0.26 \\
\hline Hyperlipidemia, $n(\%)$ & $20(24.4)$ & $122(24.1)$ & 0.95 \\
\hline Alcohol consumption, $n(\%)$ & $5(6.5)$ & $13(2.5 \%$ & 0.06 \\
\hline Current cigarette smokers, $n(\%)$ & $24(29.3)$ & $73(14.2)$ & $<0.001$ \\
\hline Former cigarette smokers, $n(\%)$ & $18(22.0)$ & $73(14.2)$ & \\
\hline Lifelong nonsmokers, $n(\%)$ & $40(48.8)$ & $367(71.5)$ & \\
\hline NIHSS at the time of admission & $6.5(3.0-22.0)$ & $6.0(3.0-17.7)$ & 0.65 \\
\hline Intracerebral hemorrhage, $n(\%)$ & $12(14.6)$ & $60(11.7)$ & 0.26 \\
\hline Ischemic stroke, $n(\%)$ & $63(76.8)$ & $431(84.0)$ & \\
\hline Subarachnoid hemorrhage, $n(\%)$ & $4(4.9)$ & $10(1.9)$ & \\
\hline Undefined stroke, $n(\%)$ & $3(3.7)$ & $12(2.3)$ & \\
\hline
\end{tabular}

According to the patients' neighborhood, patients were categorized into 3 subgroups: less privileged area, middle privileged area, and high privileged area. NIHSS, National Institutes of Health Stroke Scale; SUD, substance use disorders; SUD group, patients with current use or history of SUD; non-SUD group, patients without any history of SUD. All variables were shown as number (percentage) except the following: ${ }^{\dagger}$ Mean \pm SD. ${ }^{\ddagger} \mathrm{Me}-$ dian (Q1-Q3).

\section{Statistical Analysis}

Data were expressed as means \pm SD or median (interquartile range) according to the pattern of data distribution. We used the $\chi^{2}$ test to analyze categorical variables. Group comparisons were performed using the sample $t$ test (for normally distributed data) or Mann-Whitney test (for nonnormally distributed data). Using binary univariable and multivariable logistic regression models (backward stepwise method), we analyzed the odds ratios of poststroke severity, disability, and functional dependency, stroke among substance users compared to the non-SUD group. The cumulative rates of mortality were compared using the log-rank test. Using multivariable Cox regression models, we calculated the adjusted hazard ratio (aHR) of SUD on poststroke mortality at 7 days, 1 month, 3 months, 1 year, and 5 years after stroke. Variables with a significant or borderline $p$ value $(p<0.1)$ in univariable analyses were entered into a multivariable regression model [28]. We used competing risk analysis to adjust the possible effect of death on the recurrence rate and estimated subdistribution hazard ratio of recurrence. A two-sided $p<0.05$ was considered statistically significant. All statistical analyses were performed using SPSS for Windows TM, version 25, software package (SPSS Inc., Chicago, IL, USA) and Stata (version 13).

\section{Results}

Data regarding stroke and SUD were available for 595 patients with FES (mean age of $63.5 \pm 16.0$ years). Eightysix $(14.5 \%)$ patients had a hemorrhagic stroke, 494 (83.0\%) had an ischemic stroke, and 15 (2.5\%) had a stroke with undefined etiology. Table 1 summarizes demographic and socioeconomic differences between these SUD versus non-SUD groups. Eighty-two (13.8\%) patients were current substance users including opium $(n=$ $68,82.9 \%)$, naswar $(n=5,6.1 \%)$, hashish $(n=1,1.2 \%)$, and heroin $(n=1,1.2 \%)$. Seven (8.5\%) patients used other types of substances or a combination of them. SUD was more frequent in men than women (M/F: $2.42, p<0.001)$. The rate of vascular risk factors was similar between the SUD and non-SUD groups, except for a higher rate of cigarette smoking in the SUD group $(p<0.001)$. 


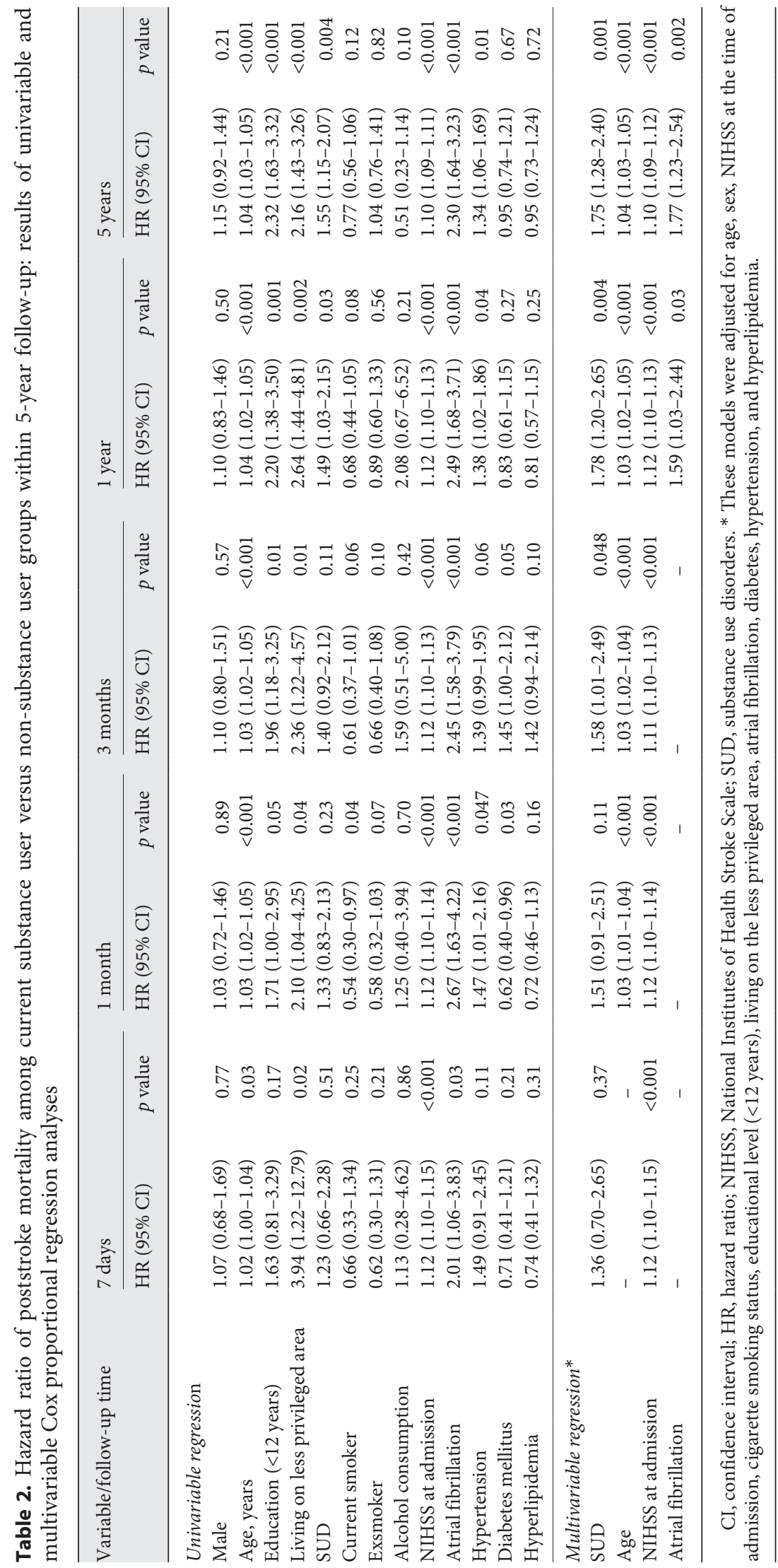




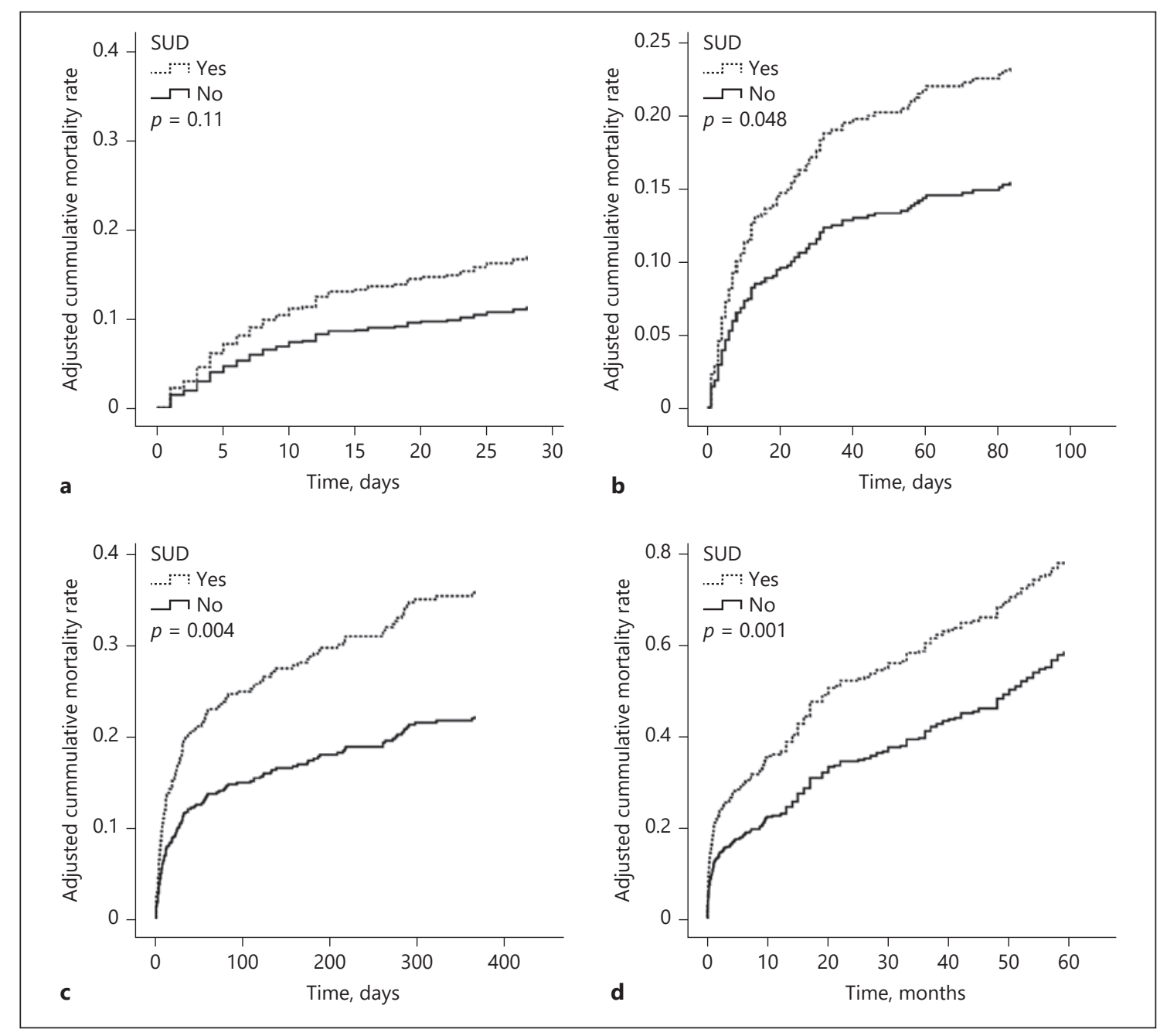

Fig. 1. Adjusted cumulative mortality rate among current substance user versus non-substance user groups within 5-year follow-up: at 1 month (a), 3 months (b), 1 year (c), and 5 years (d) after stroke index. SUD, substance use disorder.

\section{Clinical Outcomes}

During the study period, the cumulative rates of mortality among current substance users versus the non-SUD group were significantly different at 1 -year $(0.43$ vs. 0.31 ; $p=0.03)$ and 5 -year follow-ups ( 0.72 vs. $0.55 ; p=0.003)$. After adjustment for covariates including age, sex, sociodemographic data (educational level $<12$ years and living in the less privileged area), vascular risk factors (cigarette smoking, alcohol consumption, atrial fibrillation, diabetes, hypertension, and hyperlipidemia), and NIHSS at admission, current substance users had a higher hazard ratio of death at 3-month (aHR: 1.58, CI: 1.01-2.49), 1-year (aHR: 1.78, CI: 1.20-2.65), and 5-year (aHR: 1.75, CI: 1.28-2.40) follow-ups as compared to the non-SUD group (Table 2; Fig. 1a-d). In multivariable binary logis- tic regression analysis, there was no significant difference in the odds ratio of stroke severity, poststroke disability, functional dependency, and recurrence among the SUD group versus non-SUD group. In addition, in competing risk analysis, we did not observe any significant difference in the risk of stroke recurrence between the SUD group versus non-SUD group (Table 3).

\section{Discussion}

In a population-based study of stroke among 595 cases with the first-ever stroke, we examined the association of substance use and long-term stroke outcomes, including mortality, recurrence, poststroke disability, and func- 
Table 3. Odds ratio of poststroke disability, functional dependency, and recurrence and severity of stroke among current substance user versus non-substance user groups within 5-year follow-up: results of the multivariable logistic regression model

\begin{tabular}{|c|c|c|c|c|c|c|}
\hline Stroke outcomes & \multicolumn{2}{|l|}{3 months } & \multicolumn{2}{|l|}{1 year } & \multicolumn{2}{|l|}{5 years } \\
\hline Disability** & $0.63(0.23-1.67)$ & 0.35 & $1.19(0.42-3.37)$ & 0.74 & $0.54(0.09-3.22)$ & 0.50 \\
\hline Functional dependency** & $0.90(0.30-2.64)$ & 0.84 & $1.49(0.48-4.59)$ & 0.49 & $0.75(0.08-7.07)$ & 0.80 \\
\hline Recurrence ${ }^{\ddagger}$ & $0.80(0.24-2.62)$ & 0.71 & $0.78(0.23-2.58)$ & 0.69 & $1.05(0.51-2.15)$ & 0.88 \\
\hline
\end{tabular}

${ }^{\dagger}$ Odds ratios for the substance use disorder group. ' Severe stroke was defined as National Institutes of Health Stroke Scale (NIHSS) $\geq 8$; this model was adjusted for age, sex, atrial fibrillation, hypertension, diabetes mellitus and hyperlipidemia, cigarette smoking status, alcohol consumption, education level ( $<12$ years), and living in the less privileged area. ${ }^{* *}$ Disability and functional dependency were defined as mRS $>2$ and Barthel Index $<60$; models were adjusted for age, sex, NIHSS at the time of admission, cigarette smoking status, alcohol consumption, education level ( $<12$ years), living on the less privileged area, diabetes, hypertension, and hyperlipidemia. ${ }^{\ddagger}$ Adjusted for age, sex, atrial fibrillation, hypertension, diabetes mellitus, hyperlipidemia, NIHSS at the time of admission, cigarette smoking status, alcohol consumption, education level ( $<12$ years), and living on the less privileged area. Subdistribution hazard ratio for the substance use disorder group.

tional dependency. After adjustment for a wide range of socioeconomic and vascular risk factors, current SUD significantly increased the hazard ratio of stroke mortality. There was no significant difference in odds of having a poststroke disability, functional dependency, severity of the stroke, and stroke recurrence between those with a history of current SUD versus their counterparts.

A large body of evidence suggests an association between different substances, including stimulants, opioids, hallucinogens, and cannabis, with stroke [1-7]. However, little is known regarding the long-term outcome of stroke among substance users. In the current study, $13.8 \%$ of patients with stroke were current substance users at the time of the index stroke. Opium was the most frequent substance of abuse $(82.9 \%)$. These findings are in line with the Iranian household Mental Health Survey (IranMHS), showing opium use disorder as the most common SUD in Iran [29]. Likewise, the rate of opium addiction among cases with stroke in Iran varied from 11.5\% [2] to $30.8 \%$ [4] and 39.9\% [30]. Despite the differences in the age of population between the MSIS and IranMHS (relatively younger population than MSIS), a higher rate of SUD in cases with stroke is worrisome, requiring the urgent attention of health authorities.

The substance may increase the risk of stroke directly via vascular damage or indirectly because of drug-associated organ lesions (i.e., heart and liver) [31]. A wide range of drug-associated mechanisms can contribute to both ischemic and hemorrhagic strokes. Acute hypertension, rupture of aneurysms, and vasculitis can lead to hemorrhagic strokes [31, 32]. Arrhythmias, hypoxia, hypotension, vasospasm, atherosclerosis, thrombus formation, and embolism may cause ischemic strokes [31, 32]. The risk, etiology, and type of stroke vary according to the type and route of drug administration [31,33], therapeutic usage $[34,35]$, and the purity of drugs [36-38]. For example, opioids may cause ischemic stroke due to cardiac dysrhythmia [39], endocarditis [31], and atherosclerosis [40]. Soroosh et al. [41] reported the association between prolongation of QTc interval, as one of the risk factors for stroke incidence [42], and opium overdose. Opioids are also associated with hemorrhagic stroke due to pyogenic arteritis, aneurysmal rupture, and eosinophilia [31]. Recent studies have suggested that impurities of illicit drugs may contribute to the long-term side effects of substances [36]. Unlike prescription opioids [34], illicit opium often contains adulterants and may transmit heavy metals (i.e., lead) [37] or microbial contaminations into the body $[4,35,37]$. Likewise, cannabis may also be associated with both types of stroke via different mechanisms [31, 32].

SUD is a major risk factor for premature mortality, reducing the average life span by 22.5 years [30]. While psychostimulants may increase poststroke mortality [8], there is not enough evidence for such associations with other substances [6]. In the current study, SUD signifi- 
cantly increased the hazard ratio of mortality at 3-month, 1 -year, and 5-year follow-ups. Therefore, we showed an increase in the mortality rate of stroke among current substance users. We are not able to comment on the exact reasons for increased mortality based on the current study. However, a higher rate of death among cases with stroke and SUD, including opioid use disorders, may be due to an acceleration of the atherosclerosis process [15, $40,43,44]$ through multiple mechanisms, including increased oxidative stress, hyperlipidemia, hyperhomocysteinemia, insulin resistance, hypercoagulable state, and decreased plasma adiponectin [15, 45-47]. The other probable reasons for the higher rate of mortality in patients with stroke and SUD are high-risk behaviors (e.g., drug intoxication, suicide, and intravenous injections) $[45,48,49]$, nonadherence to medications [50], baseline comorbidities such as respiratory diseases [49], and infections (hepatitis and HIV) [49, 50]. We also observed no significant association of 7-day and 1-month poststroke mortality with SUD. This is in agreement with recent reports indicating the nonsignificant association between opium use and in-hospital mortality from CVD [39].

In our study, an increased hazard ratio of mortality was independent of the traditional vascular risk factors and sociodemographic variables, indicating the importance of SUD as a determinant of stroke outcome. We did not find any association between SUD and stroke recurrence and disability. This finding goes against some alleged beliefs regarding the benefit of SUD and, in particular, opium on alleviating the risk factors of vascular events [14]. The public should be aware that illicit drugs, including opium, have no benefit whatsoever in improving stroke disability and may increase the chance of death.

Our study has some limitations. Collecting data regarding substance use by interviewing patients - or their next of kin - is a subject of self-report response bias or underreporting SUD. We are not able to comment regarding the amount, duration of usage before admission, and abstinence rates after discharge. We considered participants with a history of substance use as a non-SUD group if they had continued their abstinence for at least 1 year; however, there is no evidence that 1 year of abstinence is enough to eliminate all possible vascular effects of substances. This study could not reveal the underlying pathophysiological mechanisms of the observed association between stroke, poststroke mortality, and SUD due to many confounders, such as the possible effects of adulterants of illicit drugs. The major strength of our study is that its population-based design makes our sample a good representative of stroke patients in the Middle East. In addition, the rich database in the MSIS provides an opportunity to adjust our data for a wide range of traditional vascular risk factors and sociodemographic variables.

\section{Conclusion}

In summary, SUD is a strong determinant of mortality among stroke survivors. SUD, including opium, does not reduce stroke disability or functional dependency. The public should be aware of the risk of all substances and avoid them.

\section{Acknowledgements}

We are truly grateful to 980 community health volunteers for their major contribution to this study.

\section{Statement of Ethics}

The study was conducted according to the guidelines of the Declaration of Helsinki and approved by the ethics committee of the Mashhad University of Medical Sciences, Mashhad, Iran (No. 910678). Informed consent was obtained from the participants or the next of kin before any interview or neurologic examination.

\section{Conflict of Interest Statement}

The authors have no conflicts of interest to declare.

\section{Funding Sources}

The MSIS was supported by a grant from the Mashhad University of Medical Sciences, Mashhad, Iran (No. 910678).

\section{Author Contributions}

Mohammad Sobhan Sheikh Andalibi: reporting of the work described in the article, manuscript revision, data analysis, and submitting the manuscript. Amir Rezaei Ardani, Mahmoud Reza Azarpazhooh, and Naghmeh Mokhber: planned and supervised the study, reporting of the work described in the article, and revised the manuscript. Amin Amiri: conducted the study and reporting of the work described in the article. Negar Morovatdar: reporting of the work described in the article and data analysis. Amirhossein Talebi: reporting of the work described in the article. All authors reviewed, edited, and approved the final version of the article. 


\section{References}

1 Cheng YC, Ryan KA, Qadwai SA, Shah J, Sparks MJ, Wozniak MA, et al. Cocaine use and risk of ischemic stroke in young adults. Stroke. 2016;47(4):918-22.

2 Ebrahimi H, Haghjoo Javanmard S, Asgary S, Dehghani L, Amiri M, Saadatnia M. Opium addiction and Ischemic stroke in Isfahan, Iran: a case-control study. Eur Neurol. 2018; 79(1-2):82-5.

3 Lappin JM, Darke S, Farrell M. Stroke and methamphetamine use in young adults: a review. J Neurol Neurosurg Psychiatry. 2017; 88(12):1079-91.

4 Mousavi-Mirzaei SM, Talebi A, Amirabadizadeh A, Nakhaee S, Azarkar G, Mehrpour O. Increasing the risk of stroke by opium addiction. J Stroke Cerebrovasc Dis. 2019;28(7): 1930-5.

5 Kalla A, Krishnamoorthy PM, Gopalakrishnan A, Figueredo VM. Cannabis use predicts risks of heart failure and cerebrovascular accidents: results from the national inpatient sample. J Cardiovasc Med. 2018;19(9):480-4.

6 Esse K, Fossati-Bellani M, Traylor A, MartinSchild S. Epidemic of illicit drug use, mechanisms of action/addiction and stroke as a health hazard. Brain Behav. 2011;1(1):44-54.

7 Hackam DG. Cannabis and stroke: systematic appraisal of case reports. Stroke. 2015; 46(3):852-6.

8 Darke S, Duflou J, Kaye S, Farrell M, Lappin J. Psychostimulant use and fatal stroke in young adults. J Forensic Sci. 2019;64(5): 1421-6.

9 Whiteford HA, Degenhardt L, Rehm J, Baxter AJ, Ferrari AJ, Erskine HE, et al. Global burden of disease attributable to mental and substance use disorders: findings from the Global Burden of Disease Study 2010. Lancet. 2013;382(9904):1575-86.

10 Ganguly K, Sharma H, Krishnamachari K. An ethnographic account of opium consumers of Rajasthan (India): socio-medical perspective. Addiction. 1995;90(1):9-12.

11 Lee P. Opium culture: the art and ritual of the Chinese tradition: Inner Traditions/Bear \& Co; 2006.

12 Nazari SM, Naseri M, Mokri A, Ghaffari F, Davati A, Kamalinejad M. Opium and opium addiction treatment in persian traditional medicine. Med Hist J. 2012;3(9):133-50.

13 Zarghami M. Iranian common attitude toward opium consumption. Iran J Psychiatry Behav Sci. 2015;9(2):e2074.

14 Farahani MA, Mohammadi E, Ahmadi F, Maleki M, Hajizadeh E. Cultural barriers in the education of cardiovascular disease patients in Iran. Int Nurs Rev. 2008;55(3):360-6.

15 Najafipour H, Beik A. The impact of opium consumption on blood glucose, serum lipids and blood pressure, and related mechanisms. Front Physiol. 2016;7:436.

16 Azarpazhooh MR, Etemadi MM, Donnan GA, Mokhber N, Majdi MR, Ghayour-Mobarhan M, et al. Excessive incidence of stroke in Iran: evidence from the Mashhad stroke incidence study (MSIS), a population-based study of stroke in the Middle East. Stroke. 2010;41(1):e3-e10.

17 Farzadfard MT, Thrift AG, Amiri A, Kapral MK, Hashemi P, Sposato LA, et al. Five-year case fatality following first-ever stroke in the Mashhad stroke incidence study: a population-based study of stroke in the Middle East. J Stroke Cerebrovasc Dis. 2018;27(4):1085-9.

18 Shoeibi A, Salehi M, Thrift AG, Kapral MK, Farzadfard MT, Azarpazhooh A, et al. Oneyear case fatality rate following stroke in the Mashhad stroke incidence study: a population-based study of stroke in Iran. Int J Stroke. 2015;10:96-102.

19 Farzadfard MT, Sheikh Andalibi MS, Thrift AG, Morovatdar N, Stranges S, Amiri A, et al. Long-term disability after stroke in Iran: evidence from the Mashhad stroke incidence Study. Int J Stroke. 2019;14(1):44-7.

20 Salehi M, Amiri A, Thrift AG, Kapral MK, Sposato L, Behrouz R, et al. Five-year recurrence rate and the predictors following stroke in the Mashhad Stroke Incidence Study: a population-based cohort study of stroke in the Middle East. Neuroepidemiology. 2018; 50(1-2):18-22.

21 Saber H, Thrift AG, Kapral MK, Shoamanesh A, Amiri A, Farzadfard MT, et al. Incidence, recurrence, and long-term survival of ischemic stroke subtypes: a population-based study in the Middle East. Int J Stroke. 2017; 12(8):835-43

22 Morovatdar N, Thrift AG, Kapral MK, Behrouz R, Saber H, Amiri A, et al. Long-term outcomes of ischemic stroke of undetermined mechanism: a population-based prospective cohort. Neuroepidemiology. 2017;49(3-4): 160-4.

23 Morovatdar N, Thrift AG, Stranges S, Kapral M, Behrouz R, Amiri A, et al. Socioeconomic status and long-term stroke mortality, recurrence and disability in Iran: the Mashhad Stroke Incidence Study. Neuroepidemiology. 2019;53(1-2):27-31.

24 Morovatdar N, Di Napoli M, Stranges S, Thrift AG, Kapral M, Behrouz R, et al. Regular physical activity postpones age of occurrence of first-ever stroke and improves long-term outcomes. Neurol Sci. 2020

25 Avan A, Digaleh H, Di Napoli M, Stranges S, Behrouz R, Shojaeianbabaei G, et al. Socioeconomic status and stroke incidence, prevalence, mortality, and worldwide burden: an ecological analysis from the Global Burden of Disease Study 2017. BMC Med. 2019;17(1): 191

26 Hatano S. Experience from a multicentre stroke register: a preliminary report. Bull World Health Organ. 1976;54(5):541.

27 American Psychiatric Association. DSM-IVTR: diagnostic and statistical manual of mental disorders; the encyclopedia of cross-cultural psychology. Washington; 2000.
28 Araújo RLC, Riechelmann RP. Interpretation of results from tables, graphs, and regressions in cancer research. In: Araújo RLC, Riechelmann RP, editors. Methods and biostatistics in oncology: understanding clinical research as an applied tool. Cham: Springer International Publishing; 2018. p. 71-92.

29 Amin-Esmaeili M, Rahimi-Movaghar A, Sharifi V, Hajebi A, Radgoodarzi R, Mojtabai $\mathrm{R}$, et al. Epidemiology of illicit drug use disorders in Iran: prevalence, correlates, comorbidity and service utilization results from the Iranian mental health survey. Addiction. 2016;111(10):1836-47.

30 Hamzei-Moghaddam A, Shafa MA, Khanjani N, Farahat R. Frequency of opium addiction in patients with ischemic stroke and comparing their cerebrovascular doppler ultrasound changes to non-addicts. Addict Health. 2013; 5(3-4):95.

31 Tsatsakis A, Docea AO, Calina D, Tsarouhas K, Zamfira LM, Mitrut R, et al. A mechanistic and pathophysiological approach for stroke associated with drugs of abuse. JCM. 2019; 8(9): 1295.

32 Thanvi BR, Treadwell SD. Cannabis and stroke: is there a link? Postgrad Med J. 2009; 85(1000):80-3.

33 Fallahzadeh MA, Salehi A, Naghshvarian M, Fallahzadeh MH, Poustchi H, Sepanlou SG, et al. Epidemiologic study of opium use in pars cohort study: a study of 9,000 adults in a rural Southern area of Iran. Arch Iran Med. 2017; 20(4):205-10.

34 Khodneva Y, Muntner P, Kertesz S, Kissela B, Safford MM. Prescription opioid use and risk of coronary heart disease, stroke, and cardiovascular death among adults from a prospective cohort (REGARDS Study). Pain Med. 2016;17(3):444-55.

35 Nakhaee S, Mehrpour O. The transition of lead and microbial contamination from adulterated opium to the human body. Expert Opin Drug Metab Toxicol. 2019;15(4):259-60.

36 Alinejad S, Aaseth J, Abdollahi M, HassanianMoghaddam H, Mehrpour O. Clinical aspects of opium adulterated with lead in Iran: a review. Basic Clin Pharmacol Toxicol. 2018; 122(1):56-64.

37 Hayatbakhsh MM, Oghabian Z, Conlon E, Nakhaee S, Amirabadizadeh AR, Zahedi MJ, et al. Lead poisoning among opium users in Iran: an emerging health hazard. Subst Abuse Treat Prev Policy. 2017;12(1):43.

38 Mousavi-Mirzaei SM, Khorasani EY, Amirabadizadeh A, Nakhaee S, Baharshahi A, Rajabpour-Sanati A, et al. Comparison of blood lead concentrations in patients with acute ischemic stroke and healthy subjects. J Trace Elem Med Biol. 2020;61:126532.

39 Nakhaee S, Amirabadizadeh A, Qorbani M, Lamarine RJ, Mehrpour O. Opium use and cardiovascular diseases: a systematic review and meta-analysis. Crit Rev Toxicol. 2020; 50(3):201-12. 
40 Masoomi M, Azdaki N, Shahouzehi B. Elevated plasma homocysteine concentration in opium-addicted individuals. Addict Health. 2015;7(3-4):149-56.

41 Soroosh D, Neamatshahi M, Zarmehri B, Nakhaee S, Mehrpour O. Drug-induced prolonged corrected QT interval in patients with methadone and opium overdose. Subst Abuse Treat Prev Policy. 2019;14(1):8.

42 Soliman EZ, Howard G, Cushman M, Kissela B, Kleindorfer D, Le A, et al. Prolongation of QTc and risk of stroke: The REGARDS (REasons for Geographic and Racial Differences in Stroke) study. J Am Coll Cardiol. 2012;59(16): 1460-7.

43 Bachi K, Mani V, Jeyachandran D, Fayad ZA, Goldstein RZ, Alia-Klein N. Vascular disease in cocaine addiction. Atherosclerosis. 2017; 262:154-62.
44 Kevil CG, Goeders NE, Woolard MD, Bhuiyan MS, Dominic P, Kolluru GK, et al. Methamphetamine use and cardiovascular disease: in search of answers. Arterioscler Thromb Vasc Biol. 2019;39(9):1739-46.

45 Masoomi M, Ramezani MA, Shahriari S, Shahesmaeeli A, Mirzaeepour F. Is opium addiction a risk factor for deep vein thrombosis? A case-control study. Blood Coagul Fibrinolysis. 2010;21(2):109-12.

46 Nakhaee S, Ghasemi S, Karimzadeh K, Zamani N, Alinejad-Mofrad S, Mehrpour O. The effects of opium on the cardiovascular system: a review of side effects, uses, and potential mechanisms. Subst Abuse Treat Prev Policy. 2020;15(1):30.
47 Azarpazhooh MR, Andalibi MSS, Hackam DG, Spence JD. Interaction of smoking, hyperhomocysteinemia, and metabolic syndrome with carotid atherosclerosis: a crosssectional study in 972 non-diabetic patients. Nutrition. 2020 Nov-Dec;79-80:110874.

48 Brådvik L, Berglund M, Frank A, Lindgren A, Löwenhielm P. Number of addictive substances used related to increased risk of unnatural death: a combined medico-legal and case-record study. BMC Psychiatry. 2009; 9(1):48.

49 Scott CK, Dennis ML, Laudet A, Funk RR, Simeone RS. Surviving drug addiction: the effect of treatment and abstinence on mortality. Am J Public Health. 2011;101(4):737-44.

50 Sansone RA, Sansone LA. Alcohol/substance misuse and treatment nonadherence: fatal attraction. Psychiatry. 2008;5(9):43. 